Pure Appl. Chem., Vol. 85, No. 1, pp. 41-51, 2013.

http://dx.doi.org/10.1351/PAC-CON-12-02-12

(C) 2012 IUPAC, Publication date (Web): 7 August 2012

\title{
Electrophoretic mobility of latex nanospheres in electrolytes: Experimental challenges*
}

\author{
Claire Chassagne ${ }^{\ddagger}$ and Maria Ibanez \\ Department of Environmental Fluid Mechanics, TU Delft, Stevinweg 1, \\ 2628 CN Delft, The Netherlands
}

\begin{abstract}
The electrophoretic mobility of sulfate latex nanospheres (radius $300 \pm 10 \mathrm{~nm}$ ) was measured as a function of ionic strength for different salts. The results were obtained from two similar instruments (Malvern ZetaSizer 3000 HSa and Malvern ZetaSizer Nano) using the same dispersions, in the same conditions. The values predicted from the standard electrokinetic model for constant surface charge were in good agreement with the data over a large range of ionic strength. The influence of the protocol used to fill the cells appears to be of importance between $1-10 \mathrm{mM}$ of added monovalent salt. There, the capillary wall properties seem to influence the electrophoretic measurements, even at fast field reversal (FFR), where electroosmosis should be absent. We found that during a series of measurements with monovalent salts, it was best to fill the cell starting from high ionic strength and decreasing the ionic strength during the series. The measurements with divalent salts were not sensitive to the filling procedure.
\end{abstract}

Keywords: colloidal dispersions; electrokinetics; electrophoresis; latex nanospheres; zeta potential.

\section{INTRODUCTION}

As has been demonstrated by refs. [1-3], the mobility maximum often observed when plotting the mobility as a function of the ionic strength can be interpreted using the so-called standard electrokinetic equations, provided that it is assumed that the particle has a constant surface charge density (and not a constant surface potential). The sulfate latex nanoparticles studied here can be assumed to carry a constant negative charge irrespective of ionic strength or $\mathrm{pH}$, and we will show that the standard theory describes well the measurements for both the monovalent $(\mathrm{KCl})$ and divalent $\left(\mathrm{MgCl}_{2}\right)$ salts used.

Each measured data point is the average of at least 10 consecutive measurements, and the difference between the consecutive measurements is less than $5 \%$. We noticed, however, that the difference between two data points for the same sample can display a much larger deviation when not measured consecutively. The electrophoretic mobility (expressed here in zeta potential units, see eq. 1) could vary from -75 to $-85 \mathrm{mV}$ for $5 \mathrm{mM}$ of added $\mathrm{KCl}$, for example. This is a significant difference, considering the fact that the electrophoretic mobility (in zeta potential units) varies about $10 \mathrm{mV}$ in the range $1-10 \mathrm{mM}$ of added $\mathrm{KCl}$.

The aim of the article is to report where these differences come from. We have checked and reported the influences of (a) the measurement equipment and (b) the experimental protocol. The

\footnotetext{
* Pure Appl. Chem. 85, 1-305 (2013). A collection of invited papers based on presentations at the $32^{\text {nd }}$ International Conference on Solution Chemistry (ICSC-32), La Grande Motte, France, 28 August-2 September 2011.

${ }^{\ddagger}$ Corresponding author
} 
reported electrophoretic mobilities were measured using commercial devices that make use of laser Doppler velocimetry.

\section{EXPERIMENTAL}

\section{Particles}

The measured particles were obtained from Interfacial Dynamics Corporation. They consist of surfactant-free polystyrene sulfate latex. The radius of the particles was measured by dynamic light scattering and found to be $300 \pm 10 \mathrm{~nm}$. The radius found by transmission electron microscopy (TEM) reported by the manufacturer is $265 \mathrm{~nm}$. For the theoretical derivations, we used $300 \mathrm{~nm}$. The sulfate groups are considered to be strong acid and hence the particle's surface charge should not vary as a function of $\mathrm{pH}$ or ionic strength. The manufacturer reports that the surface charge of the particles is $3.9 \times 10^{-2} \mathrm{C} / \mathrm{m}^{2}$. This is the value used in the calculations. The particle concentration was about $19 \mathrm{mg} / \mathrm{L}$ in all experiments. We checked that the values of the electrophoretic mobilities were insensitive to particle concentration changes in the range $10-30 \mathrm{mg} / \mathrm{L}$. The temperature was set at $298 \mathrm{~K}$. The $\mathrm{pH}$ of all suspensions was about 7.5. The samples were made by adding the required amounts of particle stock solution and electrolyte solution. These electrolyte solutions were prepared from pure water (conductivity $<1 \mu \mathrm{S} / \mathrm{cm}$, PureLab) and analytical grade salts $\left(\mathrm{MgCl}_{2}\right.$ and $\left.\mathrm{KCl}\right)$.

\section{Apparatus}

Two commercial devices were used to measure the electrophoretic mobility of the samples: the Malvern ZetaSizer $3000 \mathrm{HSa}$ (ZetaSizer) and the Malvern ZetaNano ZS cell (ZetaNano). Both measuring devices use laser Doppler velocimetry to assess the particles' mobility. Details of the technique can be found in refs. [4-6]. The main differences between the measuring devices are as follows:

- In the ZetaNano, only the scattering beam passes through the capillary cell and the reference beam is routed outside. Analysis of the Doppler shift in the ZetaNano is done by using phase analysis light scattering. In the ZetaSizer, the two laser beams cross in the capillary cell and Doppler shift analysis is performed by Fourier transformation.

- The capillary cell of the ZetaSizer is made of quartz and platinum electrodes, whereas the U-shaped capillary cell used for the ZetaNano was the disposable polycarbonate "Size \& Zeta potential" Folded Capillary cell DTS1060, which has gold electrodes.

In principle, these differences should not have an influence on the measurements. We noticed, however, that for all samples we have measured so far using both the ZetaSizer and the ZetaNano we had to apply $50 \mathrm{~V}$ across the DTS1060 cell, instead of the $150 \mathrm{~V}$ advised by Malvern in order to get comparable measurements between the devices. We used an applied voltage of $150 \mathrm{~V}$ for the ZetaSizer. (For lower voltages, the signal-to-noise ratio deteriorates.) As the measurements should be independent of the applied voltage value, we also checked that the measured mobilities were insensitive to the applied voltage, around $50 \mathrm{~V}$ for the ZetaNano (around an applied voltage of $150 \mathrm{~V}$, the measurements were voltage-sensitive) and around $150 \mathrm{~V}$ for the ZetaSizer.

\section{Protocol}

Before each series of measurements the cells were thoroughly cleaned following the instrument manufacturer protocol with ethanol, and then extensively with pure water of conductivity $<1 \mu \mathrm{S} / \mathrm{cm}$. For each measurement, $10 \mathrm{~mL}$ of the suspension to be measured was injected gently with a syringe in the cells. The minimum volume required for the build-in quartz capillary cell of the ZetaSizer is $2 \mathrm{~mL}$, and it is $0.75 \mathrm{~mL}$ for the ZetaNano. The large volume injected into the cells was meant to insure that the cells 
were sufficiently washed with the desired suspensions. Except when stated otherwise, no extra cleaning was performed during a series of measurement. In order to test whether the cell wall properties were affected by the way the series of experiments were conducted, two types of experiments were performed:

- $\quad$ (high > low) series: the samples are injected starting from the sample with the highest ionic strength, and finishing with the sample with the lowest ionic strength.

- $\quad$ (low > high) series: the samples are injected starting from the sample with the lowest ionic strength, and finishing with the sample with the highest ionic strength.

For each series, the same samples were used, only the filling order was changed. We checked for all samples that the conductivity measured in the cells corresponds to the conductivity expected for the given salt concentration. Below $0.05 \mathrm{mM}$ of added salt (below $0.02 \mathrm{mS} / \mathrm{cm}$ ), however, the conductivities displayed by the measuring devices were not reliable anymore. The measurements were run simultaneously with the ZetaSizer and the ZetaNano. The two series were measured the same day. Each data point presented in the figures is the average of more than 10 consecutive measurements. The difference between each value measured within these consecutive measurements is less than $5 \%$.

\section{THEORY AND MODELS}

The measured mobilities are plotted as an "apparent" zeta potential obtained from the Smoluchowski formula, as is often done in the literature. This Smoluchowski zeta potential $\zeta_{\text {Smolu }}(\mathrm{V})$ is linked to the particle mobility $u$ through

$$
\zeta_{\text {Smolu }}=u \eta /\left(\varepsilon_{1} \varepsilon_{0}\right)
$$

where $\eta=8.904 \times 10^{-4} \mathrm{~Pa} \cdot \mathrm{s}$ is the viscosity of the solvent (water) and $\varepsilon_{1} \varepsilon_{0}=80 \times 8.8542 \times 10^{-12}(\mathrm{~F} / \mathrm{m})$ its dielectric permittivity. $u\left(\mathrm{~m}^{2} / \mathrm{Vs}\right)$ is the particle electrophoretic mobility, which is related to the particle's velocity $v(\mathrm{~m} / \mathrm{s})$ by $v=u E$ where $E(\mathrm{~V} / \mathrm{m})$ is the applied electric field.

\section{Relation between surface charge and surface potential}

The relation between the particle's surface charge and surface potential is evaluated numerically using the following equations:

(1) Poisson-Boltzmann equation:

$$
\nabla^{2}\left(\frac{e \Psi_{\mathrm{eq}}}{k T}\right)=-\kappa^{2} \sum_{i} z_{i} v_{i} \exp \left(\frac{-z_{i} e \Psi_{\mathrm{eq}}}{k T}\right) / \sum_{i} z_{i}^{2} v_{i}
$$

where $\Psi_{\text {eq }}$ is the electric potential around the particle in the absence of an external applied electric field for which we have defined $\Psi_{\text {eq }}(\infty)=0$ and $\Psi_{\text {eq }}=\Psi_{0}$ at the surface of the particle. The relation between surface potential and zeta potential is given by

$$
\zeta=\Psi_{\text {eq }}\left(x_{\mathrm{s}}\right)
$$

where $x_{\mathrm{s}}$ is a given distance from the particle's surface. When not specified, we will use

$$
\zeta=\Psi_{\mathrm{eq}}\left(x_{\mathrm{s}}=0\right)=\Psi_{0}
$$


Furthermore, $k=1.38 \times 10^{-23}(\mathrm{~J} / \mathrm{K})$ is the Boltzmann constant, $T=298(\mathrm{~K})$ is the temperature, $e=1.6 \times 10^{-19}$ (C) the absolute value of the electron charge, $z_{i}$ the valence of ion $i$ and $v_{i}$ its stoichiometric coefficient. $\kappa^{2}$ (where $\kappa^{-1}$ is the Debye length) is given by

$$
\kappa^{2}=\frac{-e^{2} N_{\mathrm{A}} C_{\mathrm{s}}}{\varepsilon_{0} \varepsilon_{1} k T} \sum_{i} z_{i}^{2} v_{i}
$$

where $N_{\mathrm{A}}=6.02 \times 10^{23}\left(\mathrm{~mol}^{-1}\right)$ is Avogadro's number and $C_{\mathrm{S}}$ the added salt concentration in $\mathrm{mM}$ (millimol/L).

(2) The relation between the particle surface charge $\sigma\left(\mathrm{C} / \mathrm{m}^{2}\right)$ and potential is given by Gauss' law

$$
\sigma=-\varepsilon_{0} \varepsilon_{1}\left(\frac{d \Psi_{\mathrm{eq}}}{d r}\right)_{\text {at the particle surface }}
$$

where we neglect the contribution of the particle's internal field [7].

The potential $\Psi_{0}$ corresponding to $\sigma=3.9 \times 10^{-2} \mathrm{C} / \mathrm{m}^{2}$ (the value given by the manufacturer) is found for each ionic strength by bisection method, starting from two values $\Psi_{0}^{1}$ and $\Psi_{0}^{2}$ for which $\sigma\left(\Psi_{0}^{1}\right)<\sigma<\sigma\left(\Psi_{0}^{2}\right)$ until $\left|\Psi_{0}^{1}-\Psi_{0}^{2}\right|<10^{-4}$. The relation between the dimensionless surface potential $e \Psi_{0} / \mathrm{kT}$ and ionic strength is shown in Fig. 1 for the particles used in this study. For comparison, we included the formula derived for plate-like particles for symmetric electrolytes

$$
\frac{e \Psi_{0}}{k T}=2 \operatorname{arcsinh}\left(\frac{e \sigma}{2 \varepsilon_{1} \varepsilon_{0} k T \kappa}\right)
$$

Note that the plate-like particles and the numerical solution are in very good agreement, even for small $\kappa a\left([\mathrm{KCl}]<10^{-3} \mathrm{mM}\right)$, where $a$ is the radius of the particle.

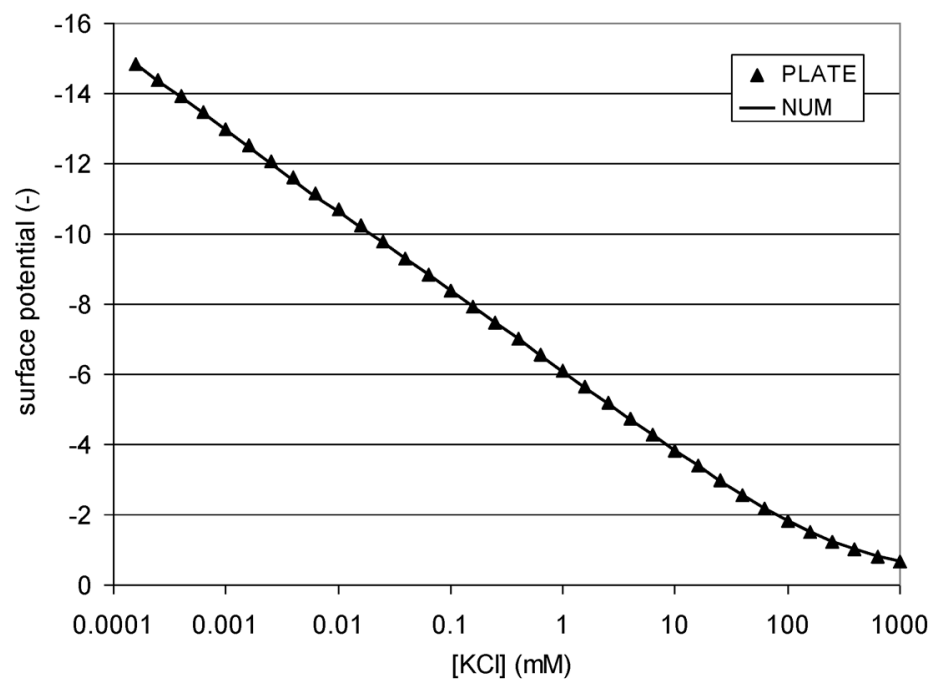

Fig. 1 Variation of the dimensionless surface potential as a function of added $\mathrm{KCl}$ concentration (mM), for a constant surface charge of $3.9 \times 10^{-2} \mathrm{C} / \mathrm{m}^{2}$. The numerical calculation (NUM) was done for a sphere of radius $300 \mathrm{~nm}$. PLATE indicates the analytical formula for flat surfaces was used. 


\section{Relation between surface charge and mobility}

For each ionic strength, a surface potential is found according to the procedure described in the preceding section. Using this surface potential, the mobility of the particle is obtained by solving numerically the standard set of electrokinetic equations (see, e.g., [7] for more details about the derivations and corresponding curves referred to as NUM in the figures' legend). Even though the numerical procedures we used are different from the procedures used by O'Brien and White and DeLacey and White, they lead to similar results. The calculated mobility differs from the Smoluchowski formula by a parameter $f$ that is often called Henry's function [7]

$$
\zeta=\zeta_{\text {Smolu }} / f
$$

In Fig. 2, the parameter $f$ is displayed (symbol $\mathrm{F}$ in the figure). The formula of Henry (valid for low zeta potentials, i.e., $e \zeta / k T<1$ ) is included for comparison. From Fig. 1 it can be seen that $e \zeta / k T<1$ for $[\mathrm{KCl}]>100 \mathrm{mM}$, where Henry's function is in agreement with the numerically evaluated parameter $f$. We also included the parameter $f$ evaluated from Ohshima's analytical solution [7], valid for $\kappa a \geq 30$

$$
\begin{aligned}
& f_{\text {Ohshima }}=1-2 \frac{2 G}{1+G} \ln \left(\frac{1+\exp |e \zeta / 2 k T|}{2}\right) /|e \zeta / 2 k T| \\
& G=\frac{2}{\kappa a}\left(1+\frac{2 \varepsilon_{1} \varepsilon_{0} k T N_{\mathrm{A}}}{\eta \Lambda^{0}}\right)(\exp |e \zeta / 2 k T|-1)
\end{aligned}
$$

where $\Lambda^{0}$ is the limiting conductance of the ion. We took $\Lambda^{0}\left(\mathrm{~K}^{+}\right) \simeq \Lambda^{0}\left(\mathrm{Cl}^{-}\right) \simeq 75 \times 10^{-4}\left(\mathrm{~S} \mathrm{~m}^{2} \mathrm{~mol}^{-1}\right)$. As can be seen in Fig. 2, Ohshima's formula indeed is valid for $[\mathrm{KCl}]>1 \mathrm{mM}$, where $\kappa a=31$.

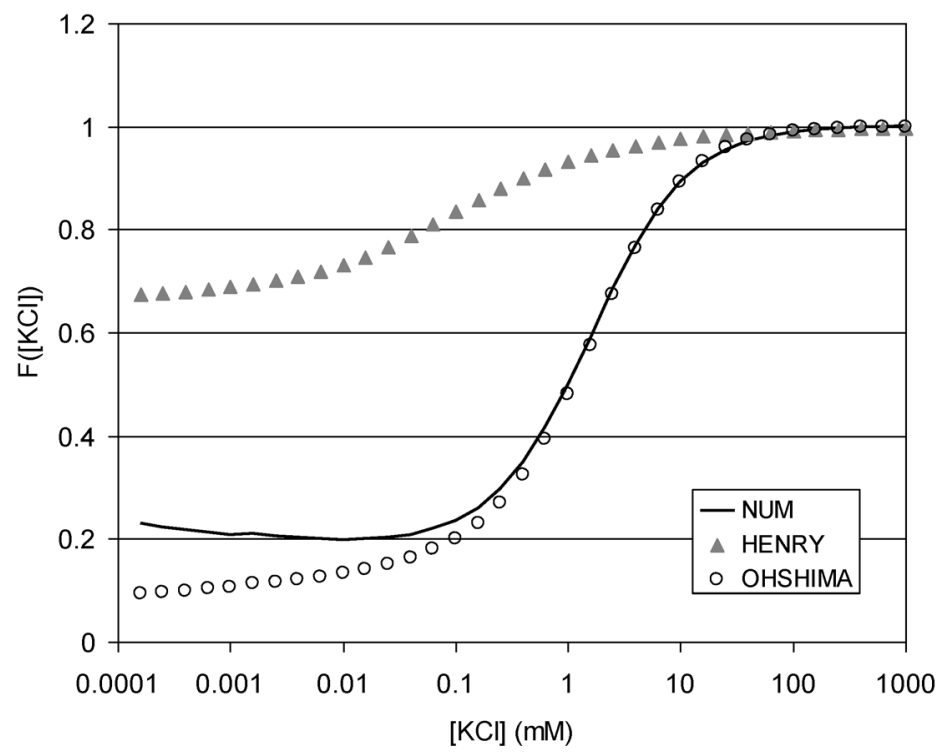

Fig. 2 Variation of the dimentionless parameter $F$ as function of added $\mathrm{KCl}$ concentration (mM) for the particle used in this study. 


\section{RESULTS AND DISCUSSION}

Monovalent salt (KCl)

\section{Influence of the measurement equipment}

There are minor differences between the results found with the ZetaSizer and the ZetaNano for each of the protocols used. In Fig. 3, we report, for example, the variation of the electrophoretic mobility found for the (high $>$ low) series. The differences between the points measured with the ZetaNano and ZetaSizer are less than $5 \%$ (the same difference as between consecutive measurements). When we used a different cell for the ZetaNano, using the same sample, we also find differences of less than $5 \%$.

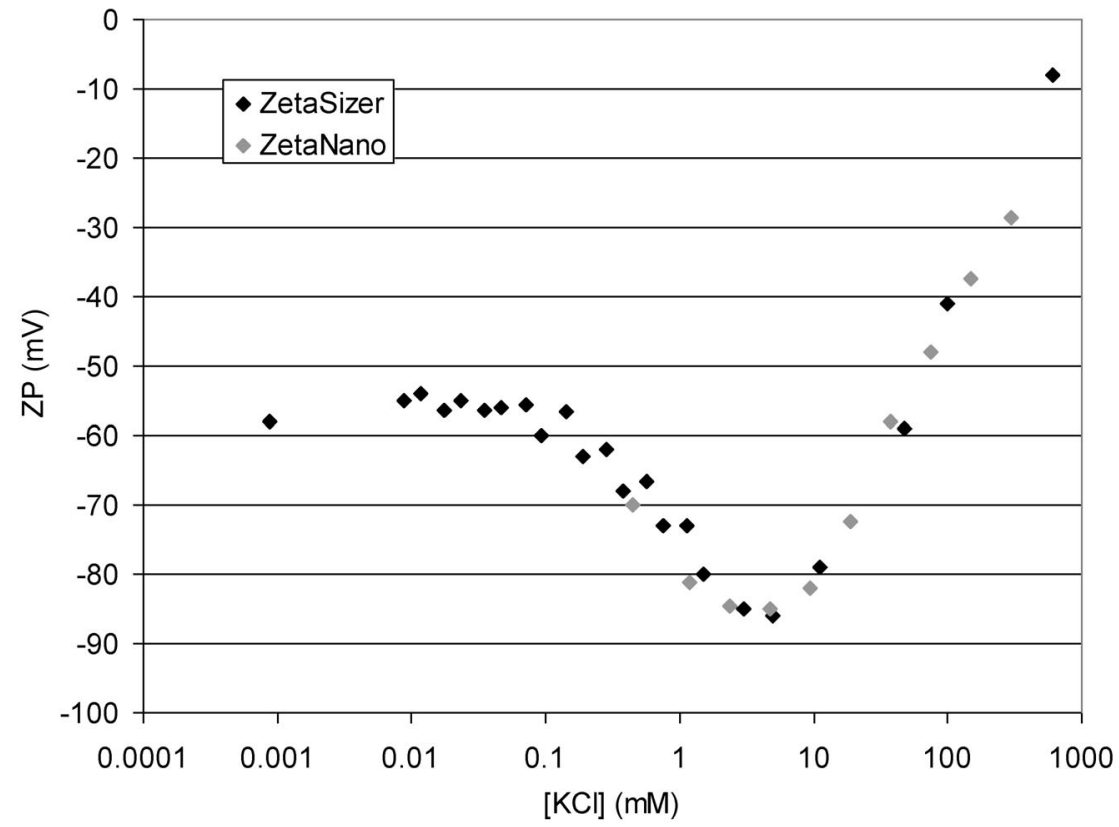

Fig. 3 Electrophoretic mobility of the latex particles of radius $300 \mathrm{~nm}$ expressed as apparent zeta potentials (ZPs) (see eq. 1), as function of added $\mathrm{KCl}$ concentration $(\mathrm{mM})$ for the two measuring equipment used in this study (high $>$ low series).

\section{Influence of the measurement protocol}

We note a significant difference (both for the ZetaSizer and ZetaNano) according to the measurement protocol. As can be observed in Figs. 4 and 5, the measured mobility expressed in zeta potential units (see eq. 1) is about $-75 \mathrm{mV}$ for the (low $>$ high) series and about $-85 \mathrm{mV}$ for the (high $>$ low) series for $5 \mathrm{mM}$ of added $\mathrm{KCl}$. This is a significant difference, considering the fact that the electrophoretic mobility (in zeta potential units) varies about $10 \mathrm{mV}$ in the range $1-10 \mathrm{mM}$ of added $\mathrm{KCl}$.

In Figs. 4 and 5, the measured mobilities are compared to the mobilities evaluated from the numerical derivations and using Ohshima's relationship and the plate-like formula

$$
\zeta_{\text {Ohshima }}(\mathrm{mV})=2000 \frac{k T}{e} \operatorname{arcsinh}\left(\frac{e \sigma}{2 \varepsilon_{1} \varepsilon_{0} k T \kappa}\right) \frac{1}{f_{\text {Ohshima }}}
$$




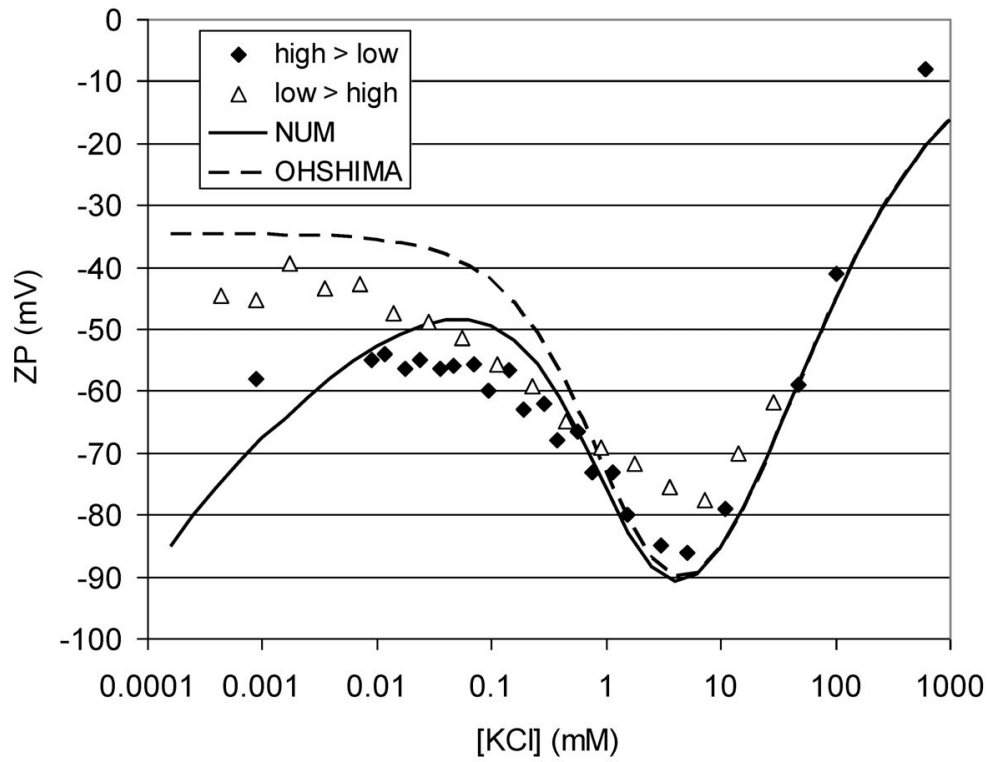

Fig. 4 Mobilities measured with the Malvern ZetaSizer 3000 HSa. The mobilities are expressed as apparent zeta potentials (ZPs), using Smoluchowski's formula. Measurement series are performed filling the cell from high to low ionic strength (high $>$ low) or from low to high ionic strength (low $>$ high). The theoretical predictions are included, using no adjustable parameters.

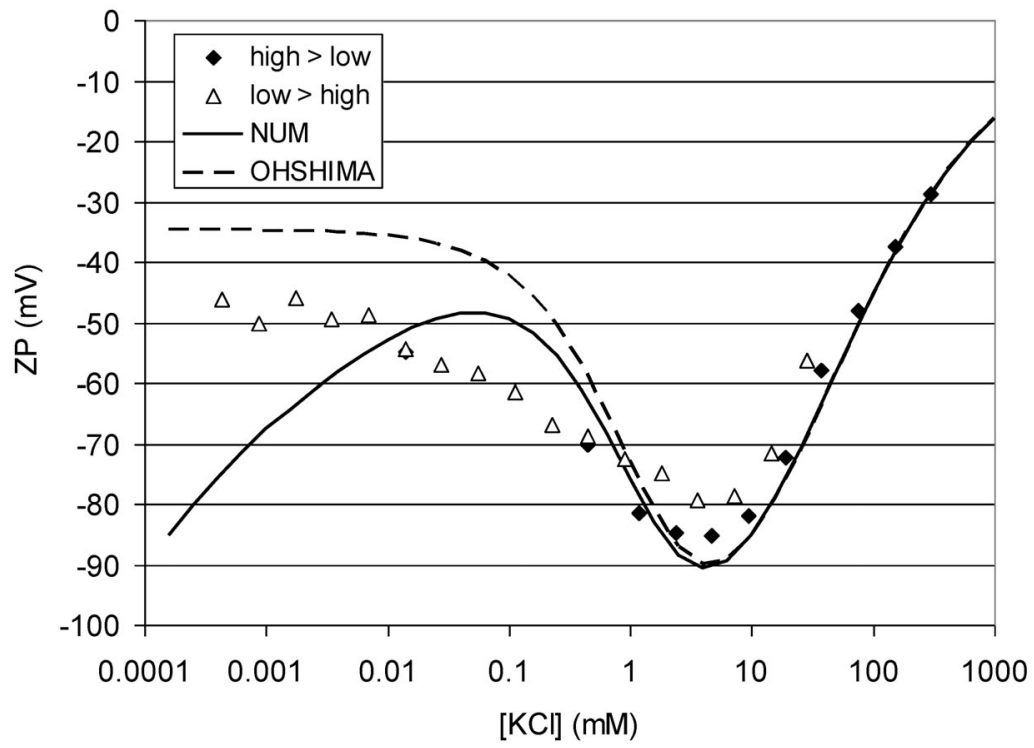

Fig. 5 Mobilities measured with the Malvern ZetaNano ZS. The mobilities are expressed as apparent zeta potentials (ZPs), using Smoluchowski's formula. Measurement series are performed filling the cell from high to low ionic strength (high $>$ low) or from low to high ionic strength (low $>$ high). The theoretical predictions are included, using no adjustable parameters. 
We note first that without any adjustable parameter, the agreement between the measured and predicted mobilities is quite good. There are, however, deviations for low values of the ionic strength, and around the mobility maximum, where the measurements are quite sensitive to the filling protocol.

Measurements at low ionic strength are difficult, as samples are very sensitive to contamination, and we found that the measurement accuracy is poor for monovalent salts (the deviations are $\pm 10 \mathrm{mV}$ for the lowest concentrations). Moreover, at concentrations around $10^{-4} \mathrm{mM}$, the double layer becomes very large $(\kappa a=0.31)$, and in theory the surface potential has to become extremely large to ensure the same constant surface charge (see Fig. 1). This could be different in practice. We could improve the agreement using either of the following hypotheses:

(i) by assuming a constant surface potential in that range of concentration, as shown in Fig. 7. In that figure, the dashed line was evaluated using a dimensionless constant surface potential of -11 (corresponding to $-275 \mathrm{mV}$ ). This is the surface potential at approximately $0.005 \mathrm{mM}$ of added $\mathrm{KCl}$, assuming the constant charge behavior (see Fig. 1).

(ii) From separate conductivity measurements, we found that below $0.005 \mathrm{mM}$ of added $\mathrm{KCl}$, the conductivity of the samples did not vary anymore and was about $0.8 \mu \mathrm{S} / \mathrm{cm}$. We could, therefore, also assume that below that concentration, the surface charge (as the surface potential) does not change anymore, as the amount of (monovalent) ions is constant. Subsequently, the electrophoretic mobility would be constant and equal to the mobility at $0.005 \mathrm{mM}$ of added $\mathrm{KCl}$. As the particles are assumed to have a constant surface charge, independent of ionic strength, we privilege the last hypothesis.

Finally, we note that for very low ionic strengths, the mobility evaluated from Ohshima's formula reaches a constant value, independent of the surface charge, equal to

$$
\zeta_{\text {Ohshima }}=-2 \ln (2) \frac{k T}{e}=-35 \mathrm{mV}
$$

Around the mobility maximum (around $5 \mathrm{mM}$ of added $\mathrm{KCl}$ ), there are significant differences between the measurement series. They were observed very systematically with the two measuring devices, and the mobility maximum was in both cases higher (in absolute value) for the (high $>$ low) series. This is also the series that corresponds best to the model in both cases. As specified in the previous section, the same samples were used for each series. The conductivity was also the same for both measuring devices at each concentration of the series, and this conductivity corresponded to the expected theoretical value. We therefore conclude that the observed differences must lay in the properties of the capillary cells and the particle/wall interactions.

To investigate the sensitivity of the measurements dependence on cell filling history we did a series of experiments where the cell was cleaned with pure water prior to each filling during a series. We then observed that the measured zeta potentials were lower (in absolute values) than the (low > high) results. This occurs when the measurements are done right after filling the cell (which takes 1 or $2 \mathrm{~min}$ ). When the cell is left to equilibrate for a longer period, from $15 \mathrm{~min}$ to $24 \mathrm{~h}$, we observed large shifts in the values of the zeta potential, especially at low ionic strength. At moderate ionic strength, cells filled using the (high $>$ low) protocol give in general time-independent results. Cells filled using the (low > high) protocol or which were cleaned prior to filling were in general time-sensitive. The filling history of the cell, and not only the equilibrium time, is therefore of importance for the capillary wall properties.

\section{Wall zeta potential}

The software of ZetaNano gives access to the so-called wall zeta potential, i.e., the zeta potential of the capillary tube in which the samples are injected [6]. This wall zeta potential is derived from the two types of measurements performed within a measurement cycle: first, the particle mobility is evaluated 
at a slow field reversal (SFR), then at a fast field reversal (FFR). At SFR, the mobility of the particle is the sum of its electrophoretic mobility and the electroosmotic velocity of the fluid (which is set in movement by the ions of capillary double layer). At FFR, the electroosmotic flow is assumed negligible and the measured mobility is the electrophoretic mobility alone. The zeta potential of the capillary walls is then evaluated by

$$
\zeta_{\text {wall }}(\mathrm{mV})=-[u(\mathrm{SFR})-u(\mathrm{FFR})] 1000 \eta /\left(\varepsilon_{1} \varepsilon_{0}\right)
$$

This wall zeta potential, therefore, gives an indication about the capillary cell wall properties: if particles are coated on it, the wall zeta potential values will be affected. The wall zeta potential corresponding to the measurements presented in Fig. 5 is given in Fig. 6. There was no significant deviation of the wall zeta potential values between the series. When we cleaned the cell with water prior to filling, the wall zeta potential was much lower (in absolute values) than these series. The capillary cell of the Malvern ZetaSizer is made of quartz, whereas the capillary cells of the ZetaNano (Size \& Zeta potential Folded Capillary cell DTS1060) are made of polycarbonate. Studies have demonstrated that latex particles have an interaction with both quartz and polymers [8-10] and might adhere to the capillary cell walls through hydrophobic forces in particular. We included the numerical predictions for the wall zeta potential using the Poisson-Boltzmann formulation for plates and assuming that the cell's surface charge was $-2.0 \times 10^{-2} \mathrm{C} / \mathrm{m}^{2}$ and that the shear plane was located at either 0.5 or 0.6 times the Debye length from the surface. Other values for the surface charge and shear plane location did not fit the experimental data. Further study is required to comment on these values and their physical interpretation.

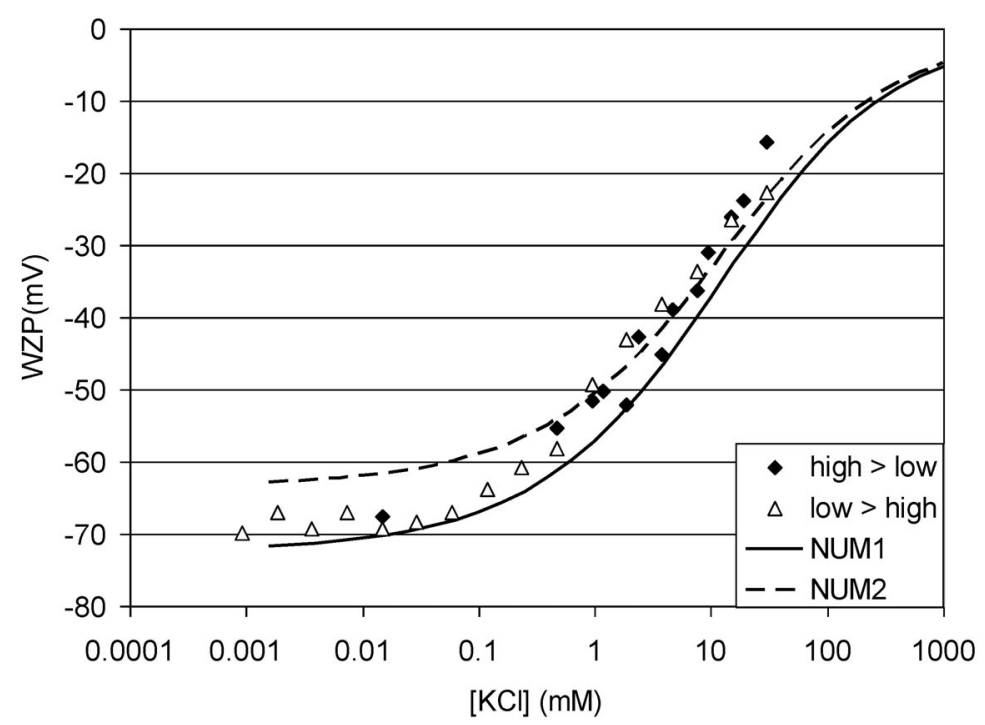

Fig. 6 Wall zeta potential (WZP) corresponding to the measurements displayed in Fig. 4. NUM1 corresponds to numerical calculations using a WZP defined at a distance of $0.5 \kappa^{-1}$ of the capillary surface and NUM2 calculations using a WZP defined at a distance of $0.6 \kappa^{-1}$ of the capillary surface. For both a constant surface charge of $-2.0 \times 10^{-2} \mathrm{C} / \mathrm{m}^{2}$ is used.

The mobility $u(\mathrm{FFR})$ is used to evaluate the particle's mobility displayed in the figures. As $u$ (FFR) is different for the different filling procedures, for the same ionic strength in the range $1-10 \mathrm{mM}$ of added $\mathrm{KCl}$, we assume that in that case electroosmosis plays a role, despite the FFR mode, and that the capillary wall properties influence the value of $u(\mathrm{FFR})$. 


\section{Divalent salt $\left(\mathrm{MgCl}_{2}\right)$}

The values measured with divalent salts are in general much more reproducible than the ones measured with monovalent salts. We also did not observe any differences between (low $>$ high) or (high $>$ low) filling protocols. The measured zeta potentials together with the numerical predictions are displayed in Fig. 7. We first tried to improve the fitting of the curve by shifting the plane of shear from the particle's surface and locate it at $x_{\mathrm{S}}=0.25$ or $0.5 \mathrm{~nm}$ from the surface, using a surface charge of $-3.9 \times 10^{-2} \mathrm{C} / \mathrm{m}^{2}$. We found that locating the shear plane at $0.25 \mathrm{~nm}$ improves the fitting. This was also found by Kobayashi, who used the same type of sulfate latex particles as we used, but of radius $1.4 \mu \mathrm{m}$ and surface charge $-7.0 \times 10^{-2} \mathrm{C} / \mathrm{m}^{2}[1]$.

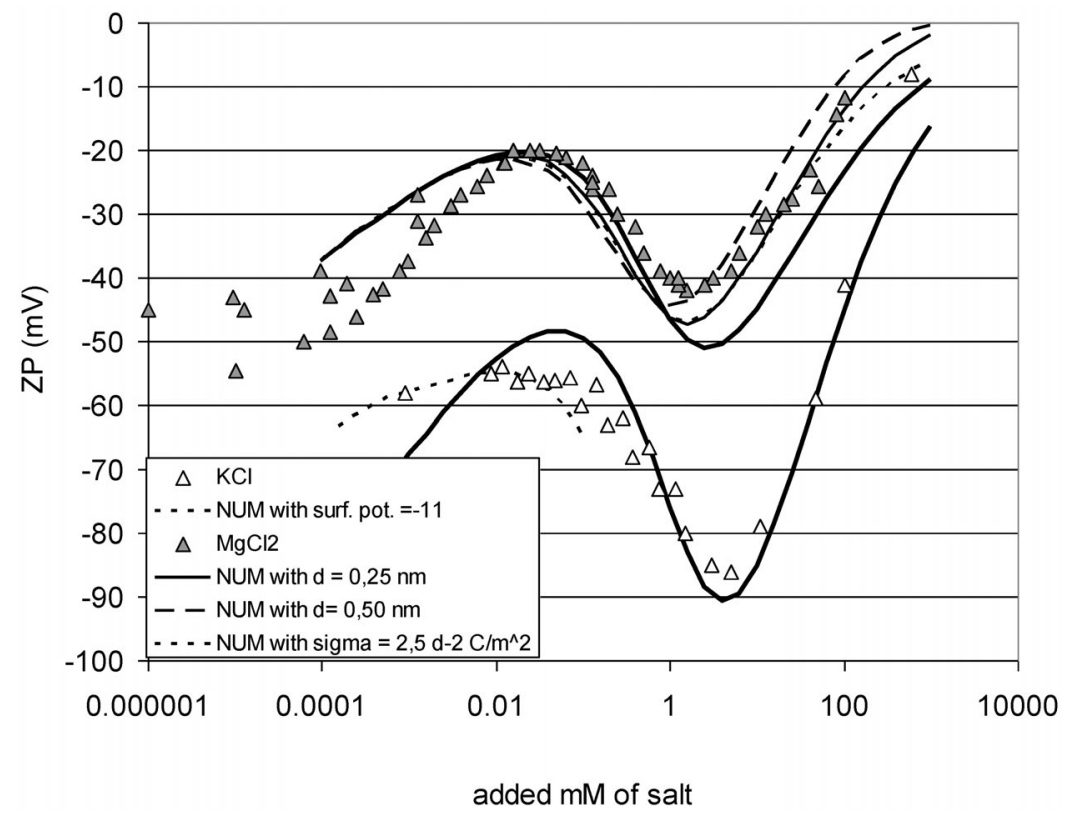

Fig. 7 Mobilities measured with the Malvern ZetaSizer 3000HSa. The mobilities are expressed in apparent zeta potentials (ZPs), using Smoluchowski's formula. Measurement series are performed filling the cell from high to low ionic strength (high $>$ low). The theoretical predictions are included, see text for details. The thick lines (not indicated in the figure's legend) were evaluated with $d=0$ and no adjustable parameter using NUM.

We then tried to fit the data by adjusting the surface charge and keeping $x_{\mathrm{S}}=0$ and found that lowering the surface charge by a factor of 1.5 (using a surface charge of $-2.5 \times 10^{-2} \mathrm{C}^{2} \mathrm{~m}^{2}$ ) lead to almost the same prediction as shifting the shear plane of $0.25 \mathrm{~nm}$ using a surface charge of $-3.9 \times 10^{-2}$ $\mathrm{C} / \mathrm{m}^{2}$. This was also observed by Kobayashi [1]. The surface potential is then nearly similar in both cases and starts to deviate slightly above $10 \mathrm{mM}$ of added salt, which is reflected in the mobility differences observed above that concentration. (The factor $1 / f$ is identical is both cases.)

The behavior at (very) low ionic strength is more in line with the numerical predictions for divalent salts. In that region, the surface potential is evaluated assuming the constant charge of the particle is half the value of the surface potential for monovalent salts (e.g., the dimensionless surface potential $e \Psi_{0} / k T$ is 12.9 for $10^{-3} \mathrm{KCl}$ and 6.3 for $10^{-3} \mathrm{MgCl}$ ). As can be observed in the figure, the numerical predictions are also less dependent on the values of surface charge or shear plane distances in that range. The differences between measured and evaluated mobility values are difficult to comment on as the theory should be adapted for low ionic strength [11]. 


\section{CONCLUSIONS}

We have demonstrated that the standard electrokinetic model for constant surface charge could be used to predict the electrophoretic mobility values for $300 \mathrm{~nm}$ sulfate latex nanospheres. The agreement was good over a large range of ionic strength, for both monovalent and divalent salts. Measurements performed on the same type but micron-sized particles gave similar results [1] in the range $0.1-1000 \mathrm{mM}$ of added salt investigated by the author. The differences we found between measured values could be related to cell-filling procedures. There were nearly no differences between measurements performed by the two types of equipment used (ZetaNano and ZetaSizer, Malvern) provided a voltage of $50 \mathrm{~V}$ is applied with the ZetaNano (and not $150 \mathrm{~V}$ as advised by the manufacturer). The cell capillary wall properties seem to affect the particle mobility even at FFR where electroosmosis should, in principle, be absent, in the case of monovalent salts. In order to improve the accuracy of the measurement in the case of monovalent salts for this type of system, we recommend starting a series of measurement with injecting the sample with the highest ionic strength first and finishing with the sample with the lowest ionic strength. Measurements with divalent salts are nearly independent of the cell-filling procedure.

\section{ACKNOWLEDGMENT}

The authors would like to thank Deltares (Delft) for the use of the Malvern 3000 HSa and Malvern ZetaNano equipment.

\section{REFERENCES}

1. M. Kobayashi. Colloid Polym. Sci. 286, 935 (2008).

2. W. Lin, M. Kobayashi, M. Skarba, C. Mu, P. Galletto, M. Borkovec. Langmuir 22, 1038 (2006).

3. M. Borkovec, S. H. Behrens, M. Semmler. Langmuir 16, 2566 (2000).

4. R. J. Hunter. Zeta Potential in Colloid Science: Principles and Applications, Academic Press, London (1981).

5. R. J. Hunter. Introduction to Modern Colloid Science, Oxford University Press, New York (1993).

6. Malvern Instruments. http://www.malvern.com/

7. H. Ohshima, K. Furusawa (Eds.). Electrical Phenomena at Interfaces, Surfactant Science series, Vol. 76, Marcel Dekker (1998).

8. H. Matsuoka, H. Morikawa, S. Tanimoto, A. Kubota, Y. Naito, H. Yamaoka. Colloid Polym. Sci. 276, 349 (1998).

9. A. Faghihnejad, H. Zeng. Soft Matter 8, 2746 (2012).

10. N. A. Mishchuk. Adv. Colloid Interface Sci. 168, 149 (2011).

11. H. Ohshima. Chem. Eng. Sci. 61, 2104 (2006). 